The Astrophysical Journal, 343:468-480, 1989 August 1

(C) 1989. The American Astronomical Society. All rights reserved. Printed in U.S.A.

\title{
THE STRUCTURE OF IO'S THERMAL CORONA AND IMPLICATIONS FOR ATMOSPHERIC ESCAPE
}

\author{
M. E. SumMers \\ Naval Research Laboratory \\ D. F. STROBEL \\ Johns Hopkins University \\ AND
Y. L. Yung, J. T. Trauger, and F. Mills
California Institute of Technology
Received 1986 August 14; accepted 1989 January 5

\begin{abstract}
We investigate the escape of species from Io's atmosphere using a steady-state model of Io's exospheric corona and its interaction with the Io plasma torus. The corona is assumed to be spherically symmetric with the radial density and compositional structure determined by the gas kinetic temperature, critical level radius, and mixing ratios of the component species. Thermal and nonthermal escape rates are calculated and the results compared with previously estimated torus and neutral cloud supply rates for $\mathrm{O}, \mathrm{S}, \mathrm{Na}$, and $\mathrm{K}$. Both oxygen- and sulfur-dominated exospheres are considered. Atmospheric sputtering is found to be the major escape mechanism for models in which the plasma flow reaches the critical level. However, such models produce total mass-loading rates an order of magnitude larger than inferred values suggesting that either (1) the structure of the thermal corona is significantly modified by the nonthermal interaction, or (2) substantial plasma flow modification and deflection occurs in the corona at or above the critical level. Assuming that the thermal model is a correct description of the corona, a comparison of these results with the observed near-Io distribution of neutral $\mathrm{Na}$ and estimated source rates for the neutral $\mathrm{Na}$ "jets" suggests an extended $\mathrm{Na}$ coronal component. Assuming that this component is part of the thermal exosphere, we find that the observations are consistent with an O-dominated corona, an exospheric temperature $\sim 1000 \mathrm{~K}$, a 0.001 critical level mixing ratio of $\mathrm{Na}$, and a critical level radius $\sim 1.5 R_{\mathrm{Io}}$.

Subject headings: planets: magnetospheres - planets: satellites
\end{abstract}

\section{INTRODUCTION}

It is known that Io is the source of the neutral $\mathrm{Na}, \mathrm{K}, \mathrm{S}$, and $O$ clouds observed in the near-Io spatial environment and in extended regions throughout the Jovian magnetosphere (Brown 1974; Trafton 1975; Brown 1981; Durrance, Feldman, and Weaver 1983; Brown, Shemansky and Johnson 1983). Ionization of the constituents in these clouds provides a source of ions for the Io plasma torus and subsequently the entire Jovian magnetosphere (Brown, Pilcher, and Strobel 1983; Belcher 1983). Several theoretical studies have attempted to determine the supply rates of neutral oxygen and sodium to these clouds (see, e.g., Smyth and McElroy 1977; Smyth and Shemansky 1983; Smyth and Combi 1987, 1988a, 1988b) and the various estimates are listed in Table 1.

Suggested modes of mass loss from Io have been discussed by many authors over the years (Kumar 1979, 1980, 1982, 1984, 1985; Kumar and Hunten 1982; Cheng 1984; Pollack and Witteborn 1980; Summers, Yung, and Haff 1983; Summers 1985; Hunten 1985). Deducing the correct mechanism hinges on a key question: Does Io possess a "thick" and hence extended atmosphere or is the atmosphere sufficiently "thin" to allow escape processes to occur near or at Io's surface? A "thick" atmosphere would probably be composed of $\mathrm{SO}_{2}$ and its photochemical products, while a "thin" atmosphere could be dominated by chemical species sputtered directly from Io's surface (Kumar 1985; Sieveka and Johnson 1985). The issue is far from settled. The answer to the question is indeed critical because most known escape processes are more efficient at the top (i.e., in the exosphere or corona) of an extended atmosphere than at the surface. The specific physical and chemical processes which occur in the atmosphere and are important for modulating the transport of material from the surface to the exobase will also depend upon the total atmospheric surface pressure.

Most photochemical models of Io's atmosphere contain assumed specific properties (boundary conditions) for Io's surface and its atmosphere near the surface (Kumar 1979, 1980, 1982, 1984, 1985; Summers 1985). These assumed properties and the postulated physical processes occurring at the surface and in the atmosphere thus determine the radial structure of the atmosphere. This has until recently been the most viable method of modeling the atmosphere since few useful observational constraints on atmospheric properties have been available. During the past few years measurements of near-Io $(<10$ $R_{\mathrm{Io}}$ ) neutral clouds containing $\mathrm{Na}, \mathrm{O}$, and $\mathrm{S}$ have been made, and from the interpretation of these observations one can hope to infer global properties of Io's extended atmosphere/ exosphere (Trauger 1984, 1985; Schneider et al. 1987; Ballester et al. 1987).

Near-Io neutral $\mathrm{Na}$ has been detected by several observers (Goldberg, Garnau, and LaVoie 1984; Pilcher et al. 1984; Trauger 1984, 1985; Schneider et al. 1987). The Trauger (1984, 1985) observations show linear jetlike features of $\mathrm{Na}$ escaping Io at very high velocities, up to the impact velocity of corotating plasma in the Io plasma torus. It has been suggested (Trauger 1984) that these "jets" are caused by corotating $\mathrm{Na}^{+}$ 
TABLE 1

ESTIMATES OF LOSS RATES FROM IO

\begin{tabular}{|c|c|c|c|}
\hline Constituent & Rate $\left(10^{26} \sec ^{-1}\right)$ & Reference & Remarks \\
\hline \multirow[t]{3}{*}{$\mathrm{Na}(\text { slow })^{\mathbf{a}} \ldots \ldots \ldots \ldots \ldots \ldots$} & 0.2 & $\begin{array}{l}\text { Smyth and McElroy } \\
1978\end{array}$ & $\begin{array}{l}\text { Ballistic modeling of the } \\
\text { region } B \text { cloud }\end{array}$ \\
\hline & 2.0 & Pilcher et al. 1984 & $\begin{array}{l}\text { Revision of Smyth and } \\
\text { McElroy (1978) rate, for } \\
\text { spatially nonuniform loss } \\
\text { region }\end{array}$ \\
\hline & 1 & $\begin{array}{l}\text { Brown and Schneider } \\
1981\end{array}$ & $\begin{array}{l}\text { Source needed for } \\
\text { "remote } \mathrm{Na} "\end{array}$ \\
\hline \multirow[t]{2}{*}{$\mathrm{Na}(\text { fast })^{\mathrm{b}}$} & 1 & Pilcher et al. 1984 & $\begin{array}{l}\text { Source for "directional } \\
\text { features" }\end{array}$ \\
\hline & 0.1 & Trauger 1975 & “Jetlike" features \\
\hline $\mathbf{K} \ldots \ldots \ldots \ldots \ldots \ldots \ldots \ldots \ldots \ldots$ & 0.1 & Trafton 1981 & $\begin{array}{l}\text { The potassium analog of } \\
\text { the B cloud }\end{array}$ \\
\hline \multirow{4}{*}{$\mathrm{O}, \ldots \ldots \ldots \ldots \ldots \ldots \ldots \ldots$} & 100 & Brown 1981 & {$[\mathrm{O}$ I $] 6300 \AA$ emission } \\
\hline & 50 & $\begin{array}{l}\text { Smyth and Shemansky } \\
1983\end{array}$ & $\begin{array}{l}\text { Cloud model of } 6300 \AA \\
\text { emission }\end{array}$ \\
\hline & 170 & $\begin{array}{l}\text { Smith and Strobel } \\
1985\end{array}$ & $\begin{array}{l}\text { Ion physics in torus } \\
\text { (oxygen torus) }\end{array}$ \\
\hline & 74 & $\begin{array}{l}\text { Smith and Strobel } \\
1985\end{array}$ & (Sulfur torus) \\
\hline \multirow[t]{2}{*}{$\mathrm{S} \quad \ldots \ldots \ldots \ldots \ldots \ldots \ldots \ldots \ldots \ldots \ldots$} & 6 & $\begin{array}{l}\text { Smith and Strobel } \\
1985\end{array}$ & (Oxygen torus) \\
\hline & 480 & $\begin{array}{l}\text { Smith and Strobel } \\
1985\end{array}$ & (Sulfur torus) \\
\hline $\begin{array}{l}\text { Total mass of injection } \\
\quad \text { (units of } \mathrm{O} \text { atom) } \ldots \ldots \ldots\end{array}$ & 250 & Dessler 1980 & $\begin{array}{l}\text { Power dissipated in } \\
\text { magnetosphere }\end{array}$ \\
\hline
\end{tabular}

a "slow" implies a velocity on the order of Io's gravitational escape velocity of $2.5 \mathrm{~km} \mathrm{~s}^{-1}$.

b "fast" implies velocities much larger than the escape velocity, typically $\sim 20 \mathrm{~km} \mathrm{~s}^{-1}$.

undergoing charge exchange with neutral $\mathrm{Na}$ in Io's upper atmosphere, the kinetic energy of the corotating $\mathrm{Na}^{+}$remaining approximately constant during the interaction, thus producing a neutral $\mathrm{Na}$ atom with a velocity approximately the same as the initial corotating $\mathrm{Na}^{+}$. The newly created $\mathrm{Na}^{+}$ will be picked up and accelerated to corotation velocity by the convection electric field in approximately one gyroperiod. The dominant source of neutral $\mathrm{Na}$ is probably in the exospheric corona, since one expects corotating plasma penetration only in the region where the atmosphere is collisionally thin (see $\S$ IV).

The observations by Trauger $(1984,1985)$ in particular are relevant to models of charge exchange in Io's corona. The observed neutral $\mathrm{Na}$ jets appear to be linear and centered on Io. The full width at half-maximum of the jets is $2-3$ Io diameters with a source strength $\sim 10^{25} \mathrm{~s}^{-1}$. By mapping the observed near-Io cross sectional structure of the $\mathrm{Na}$ jets back to Io's atmosphere one can in principle obtain information on the distribution of $\mathrm{Na}$ in that region of Io's corona which is collisionally thin to charge exchange for $\mathrm{Na}$, and hence on the average structure of Io's uppermost atmosphere.

Schneider et al. (1987) have developed and utilized a new method of observing $\mathrm{Na}$ in Io's bound and extended atmosphere. During eclipse of Europa by Io, part of the solar radiation which is incident on Europa passes through Io's atmosphere. The observed light reflected from Europa contains absorption features which are due to scattering of solar radiation in the $\mathrm{Na} \mathrm{D}$ lines by the $\mathrm{Na}$ in Io's atmosphere. Using this method Schneider et al. (1987) have used the measured $\mathrm{Na}$ absorption as a function of radial distance from Io to infer the radial distribution of $\mathrm{Na}$. The modeled distribution was found to be consistent with a $1500 \mathrm{~K}$ exosphere and a $\mathrm{Na}$ surface number density $\sim 10^{4} \mathrm{~cm}^{-3}$. These observations put severe constraints on the abundance of $\mathrm{Na}$ in Io's extended atmosphere. The $\mathrm{Na}$ abundance near Io's surface (i.e., within $500 \mathrm{~km}$ ) is, however, poorly constrained by these observations as the spatial resolution was approximately $1.2 R_{\mathrm{Io}}$. Since Io's surface (and hence near-surface atmosphere) is much cooler $(\sim 100 \mathrm{~K})$, the local scale height is much smaller than near the exobase and a much larger density of $\mathrm{Na}$ may exist near the surface than in the exosphere.

Using the $I U E$, Ballester et al. (1987) have detected emissions due to neutral oxygen and sulfur from a region within 5 $R_{\mathrm{Io}}$ of Io. Assuming electron impact excitation as the emission mechanism, Ballester et al. deduced that if the emissions were solely from $\mathrm{O}$ and $\mathrm{S}$, then large column densities $\left(\sim 10^{16} \mathrm{~cm}^{-2}\right)$ and a low electron temperature $(<2 \mathrm{eV})$ are required to produce the observed line intensities. Thus a significant fraction of the observed emissions must come from a dense collision-dominated region of Io's atmosphere. Whether the collisional excitation is from torus electrons which have undergone substantial cooling or from electrons in a hot and dense ionosphere, the $I U E$ observations suggest that a collisionally "thick" atmosphere exists around Io.

Models of Io's exospheric corona have been constructed and discussed in recent literature (Watson 1981; Ip 1982, Sieveka 1983; Sieveka and Johnson 1982, 1984, 1985; Sieveka, McGrath-Kinally, and Johnson 1985). Watson (1981) considered the sputter generation of $\mathrm{SO}_{2}$ and $\mathrm{S}$ coronae. It was found that at sufficiently large radial distances ( 2 to $\left.4 R_{\mathrm{Io}}\right)$ from Io, the sputter-generated $\mathrm{SO}_{2}$ corona could dominate the thermal component. The possibility of a predominantly atomic oxygen exosphere and the influence this would have on the structure of the exospheric corona was not discussed. This 
possibility is significant since the scale height for $\mathrm{O}$ is 4 times that for $\mathrm{SO}_{2}$, hence the thermal component of an $\mathrm{O}$ corona will be much more extended than that for $\mathrm{SO}_{2}$.

Sieveka (1983) and Sieveka and Johnson (1982, 1984, 1985) have investigated magnetospheric plasma-ion impact on Io and considered the effects this has on molecular redistribution of condensed gas species, surface, and atmospheric sputtering, and direct collisional ejection from Io's exosphere. Assuming unattenuated torus plasma impact on Io's surface, it was found that direct escape from Io due to surface sputtering probably accounts for a small fraction of the total torus mass-supply rate. McGrath and Johnson (1987) considered sputtering from a uniform atmosphere on Io and found that collisional ejection from the sputter-produced corona may be a significant source of mass for the torus, assuming that the ion flux was unattenuated to the exobase level. We show in this paper that even if a sputter-generated corona does not exist, torus/exosphere interactions with a thermal corona alone can provide a major fraction of the necessary torus supply rate.

Detailed photochemical models of a collisionally thick $\mathrm{SO}_{2}$ atmosphere (Kumar 1979, 1980, 1982, 1984, 1985; Summers 1985 ) indicate that atomic oxygen is most likely to be the dominant constituent at high altitudes. It is also suggested by these photochemical models of the dayside atmosphere and ionosphere that high thermospheric temperatures are necessary for producing the observed large dayside ionospheric scale height (Kliore et al. 1975). These models indicate that the dayside thermospheric temperature is likely to be in the range of 800 to $1300 \mathrm{~K}$ and that the critical level may be as high as one $R_{\mathrm{Io}}$ above the surface.

McEwen and Soderblom (1983) investigated the observed properties of Io's plumes and plume deposits and have made a case for the existence of two classes of volcanos on Io. In this scenario the majority of long-lived (eruptions lasting on the order of months to years) plumes are driven by hightemperature $(T \sim 400 \mathrm{~K}) \mathrm{SO}_{2}$ gas. The other class consists of short-lived (lasting possibly hours to days) higher temperatures $(\sim 650 \mathrm{~K})$ elemental sulfur-driven plumes. The explosive injection of sulfur gas into Io's atmosphere may imply that the atmosphere is at times sulfur-dominated. Thus sulfur could for short periods of time be the dominant constituent in the corona.

Further indirect support for this type of scenario comes from models of the plasma physics in the torus (Smith and Strobel 1985). In the Smith and Strobel (1985) model of the Io plasma torus, ion and energy partitioning in the torus are governed by the ion confinement time and the neutral densities of oxygen and sulfur. By comparing model results with the EUV luminosity of the torus during the Voyager 1 encounter, Smith and Strobel (1985) inferred that the average neutral torus densities at the time of the encounter were $n_{\mathrm{S}} \sim 6$ and $n_{\mathrm{O}} \sim 30\left(\mathrm{~cm}^{-3}\right.$ $\mathrm{s}^{-1}$ ). consistent with an $\mathrm{SO}_{2}$ source, and that the plasma was dominated by oxygen ions. However, the model prediction for the torus $\mathrm{O}^{++}$density was approximately an order of magnitude larger than the Brown, Shemansky, and Johnson (1983) upper limit. The only way in which Smith and Strobel (1985) could suppress the $\mathrm{O}^{++}$density sufficiently to satisfy the Brown, Shemansky, and Johnson (1983) constraint was in the limit of a sulfur-dominated torus, where the supply of neutral sulfur exceeded that of neutral oxygen by an order of magnitude. Smith and Strobel (1985) suggested that the low $\mathrm{O}^{++}$ density was characteristic of a time period shortly after eruptions of sulfur volcanoes on Io. Thus, according to the Smith and Strobel (1985) model, there are times in which the torus is supplied primarily by neutral $O$ and other times when neutral $\mathrm{S}$ injection dominates. In light of these studies we will also investigate the properties of a S-dominated corona in $\S$ III.

Ip (1982), investigating the interaction of the plasma torus with Io's exosphere, assumed a very thin collisionally dominated component of the atmosphere (only a few hundred kilometers in thickness) and a single constituent, $\mathrm{SO}_{2}$, and found a small probability $(<0.1)$ that a corotating torus ion would be absorbed by the atmosphere beyond about $500 \mathrm{~km}$ from Io's surface. However, the photochemical models previously mentioned suggest that not $\mathrm{SO}_{2}$ but $\mathrm{O}$ and $\mathrm{S}$ are probably the dominant constituents near the exobase.

In this paper we will investigate the implications of an extended, collisionally thick atmosphere on Io. We have chosen to concentrate our attention on the thermal components of Io's corona, primarly because this topic has not been discussed adequately in the literature. We construct several parameterized models of Io's exospheric corona, relying on existing models of the collision-dominated atmosphere to suggest the range of assumed coronal parameters [i.e., $\left.r_{c}, T_{c}, f_{i}\left(r_{c}\right)\right]$ and investigate atmospheric escape and the interaction of the corona with the corotating torus plasma.

The plan of this paper is as follows. In $\S$ II we describe the details of the model and the principle assumptions. Section III contains numerical results for various assumed model parameters. Several cases are presented which illustrate a range of physical properties in Io's extended corona. In $\S$ IV we discuss the implications for atmospheric escape processes. Section V concludes with a discussion and summary of this work.

\section{MODEL OF IO'S EXOSPHERIC CORONA}

The physical model of Io's exospheric corona developed here is based on the formalism developed by Chamberlain (1963) for the study of planetary coronae. In our application of this theory to Io's exosphere, we make several simplifying assumptions.

1. We assume that the gravitational field about Io is spherically symmetric. Thus we ignore the tidal interaction with Jupiter which produces inner and outer Lagrangian points at $5.79 R_{\mathrm{Io}}$ from Io's center along a line joining Io and Jupiter. We also ignore centrifugal effects due to Io's rotation.

2. We assume that Io's atmospheric structure (density, composition, and temperature) is spherically symmetric. We ignore day-night and upstream-downstream effects. The magnitude of these effects may indeed be large as discussed by Summers (1985).

3. We assume that nonthermal escape processes acting in Io's corona produce no significant perturbation on the structure of the thermal component of the corona. This issue will be examined in more detail in $\S$ IV.

4. We assume that the distribution of the dominant constituent determines the location of the critical level (see below), and that the critical level is sharply defined.

The critical level (also referred to as the exobase) is defined by the condition that the radial column density above this level, $r_{c}$, corresponds to one collision mean free path for an atom. Following Chamberlain (1963) this condition occurs when

$$
\int_{r_{c}}^{\infty} n(r) Q d r=1,
$$

where $n(r)$ and $Q$ are the dominant constituent density and 
collision cross section, respectively. From a fit to the temperature dependence of the transport coefficients determined by Konowalow, Hirschfelder, and Linder (1959) we obtain $Q_{\mathrm{o}}=1.01 \times 10^{-14} T^{0.229} \mathrm{~cm}^{2}$, and $Q_{\mathrm{s}}=6.88 \times 10^{-14} T^{0.347}$ $\mathrm{cm}^{2}$.

Above the critical level there is a relaxation of collisions and the probability of an atom colliding with another atom depends on the atom's orbital parameters. For $r>r_{c}$ the spatial and velocity distribution is determined by the particle's continuity equation in phase space (Liouville's equation) and the equations of motion. The radial density distribution of atoms is given by the modified barometric equation:

$$
n_{i}(r)=n_{i}\left(r_{c}\right) e^{-\left(\lambda_{c i}-\lambda_{i}\right)} \zeta\left(\lambda_{i}\right),
$$

where $n_{i}(r)$ is the number density of constituent $i, n_{i}\left(r_{c}\right)$ is the density at the critical level, $\lambda_{i}$ is the gravitational parameter for an atom of mass $m_{i}$, i.e.,

$$
\lambda_{i}=\frac{G M_{\mathrm{Io}} m_{i}}{k T r}
$$

$M_{\mathrm{I}}, G$, and $k$ are the mass of Io, gravitational constant, and Boltzmann constant, respectively. The partition function $\zeta\left(\lambda_{i}\right)$ is given by (see Chamberlain 1963):

$$
\begin{aligned}
\zeta(\lambda) & =\frac{1}{\sqrt{\pi}}\left\{\Gamma\left(\frac{3}{2}\right)+\gamma\left(\frac{3}{2}, \lambda\right)\right. \\
& \left.-\frac{\sqrt{\lambda_{c}{ }^{2}-\lambda^{2}}}{\lambda_{c}} e^{-\chi}\left[\Gamma\left(\frac{3}{2}\right)-\gamma\left(\frac{3}{2}, \lambda-\chi\right)\right]\right\},
\end{aligned}
$$

where

$$
\chi=\frac{\lambda^{2}}{\lambda+\lambda_{c}} .
$$

and $\Gamma$ and $\gamma$ are the complete and incomplete gamma functions, respectively.

Above the critical level the thermal component of the corona is composed of three particle populations. Ballistic particles are those which have an upward component of velocity at $r=r_{c}$, but insufficient energy to escape. Escaping particles, on the other hand, have sufficient energy to escape from the gravitational potential well. Satellite particles are those with insufficient energy to escape and orbit about the planet (Io in this case) with perigee's located at $r>r_{c}$. Thus the partition function also has three components, one for each particle population (see Chamberlain [1963] for more details).

The structure of the thermal corona is thus determined by the critical level radius $r_{c}$, gas kinetic temperature $T_{c}$, and mixing ratios $f_{i}\left(r_{c}\right)=n_{i}\left(r_{c}\right) / n\left(r_{c}\right)$. In the next section a number of models will be presented for a range of values of these parameters.

\section{NUMERICAL RESULTS}

\section{a) Oxygen-dominated Corona}

We consider here the case where atomic oxygen dominates the mass-density structure of Io's upper atmosphere. The mixing ratios of the minor constituents in Io's atmosphere are unknown. We use estimated loss rates from Io (see Table 1) as a guide for specifying the $f_{i}\left(r_{c}\right)$. We assume that the mixing ratios of the various constituents are "roughly" proportional to their

\begin{tabular}{|c|c|c|c|c|}
\hline Model $^{\mathbf{a}}$ & $R_{c}\left(R_{\mathrm{Io}}\right)$ & $T(\mathrm{~K})$ & $f_{0}\left(r_{c}\right)$ & $f_{\mathbf{S}}\left(r_{c}\right)$ \\
\hline A & 2.0 & 1000 & 0.889 & 0.1 \\
\hline B & 1.5 & 1000 & 0.889 & 0.1 \\
\hline$\ldots$ & 1.0 & 1000 & 0.889 & 0.1 \\
\hline D & 2.0 & 1500 & 0.889 & 0.1 \\
\hline E $\ldots$ & 2.0 & 500 & 0.889 & 0.1 \\
\hline ....... & 2.0 & 1000 & 0.489 & 0.5 \\
\hline $\mathrm{G} \ldots \ldots \ldots \ldots \ldots$ & 2.0 & 1000 & 0.089 & 0.9 \\
\hline
\end{tabular}
relative escape rates; we take $f_{\mathrm{K}}\left(r_{c}\right)=0.001$ and $f_{\mathrm{Na}}\left(r_{c}\right)=0.01$.
TABLE 2

Assumed Parameters for Models of Io's Thermal CORONA

${ }^{\mathrm{a}}$ In all cases $f_{\mathrm{Na}}\left(r_{c}\right)=0.01, f_{\mathrm{K}}\left(r_{c}\right)=0.001$.

Table 2 contains assumed parameters for models presented in this paper.

Model A is defined by $r_{c}=2.0 R_{\mathrm{Io}}$ and $T_{c}=1000 \mathrm{~K}$. Cases B through $\mathrm{E}$ as listed in Table 2 are presented in order to illustrate the sensitivity of coronal structure to these two param-
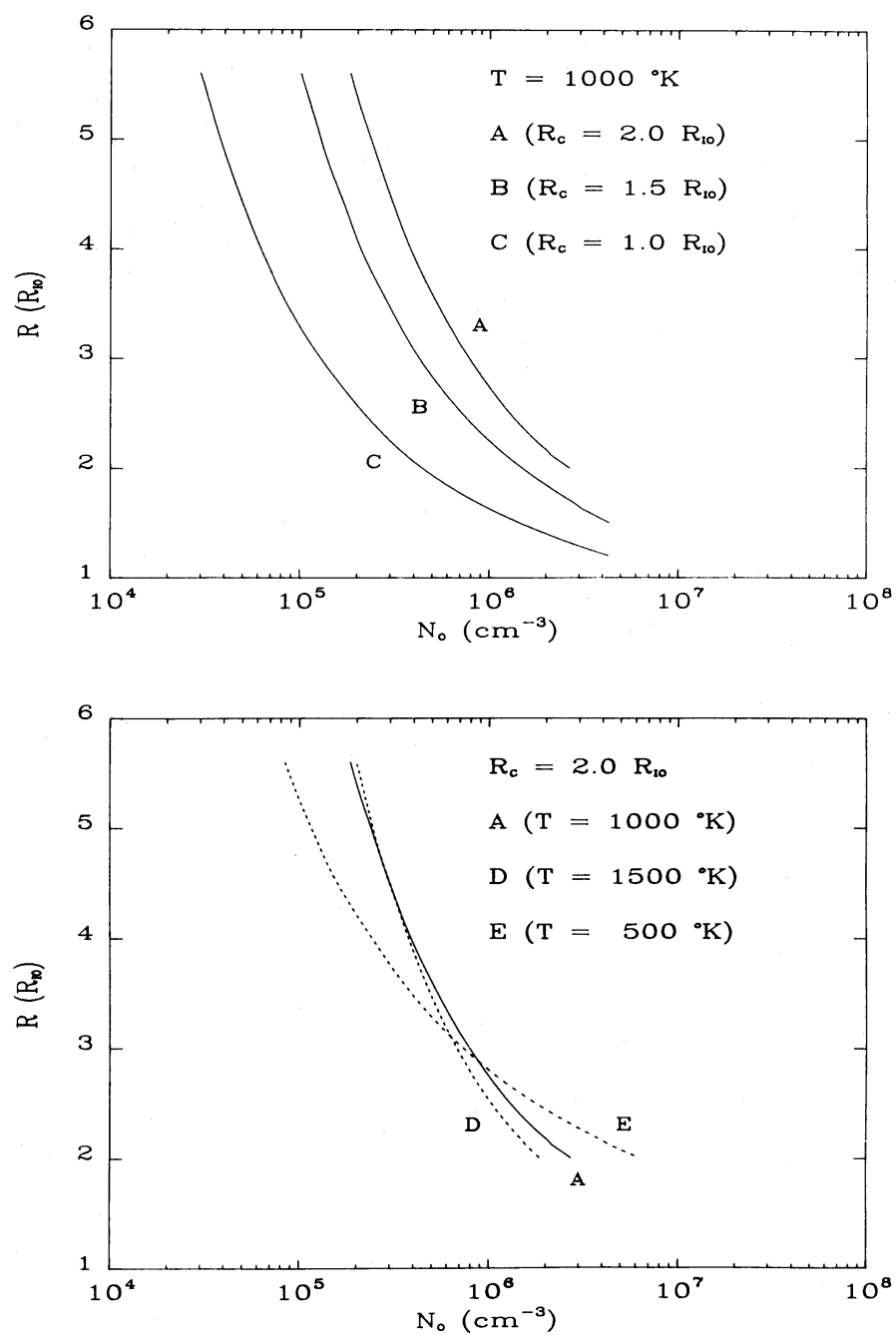

FIG. 1.- (a) Plot of the density of atomic oxygen as a function of radial distance from Io's center for the models A, B, and C in which oxygen is the major constituent in Io's corona. The radial distance is given in units of Io's radius $\left(1 R_{\mathrm{L}}=1820 \mathrm{~km}\right)$. (b) Model A, D, and E illustrating the sensitivity of the atomic oxygen density distribution to a change in assumed critical level temperature. 


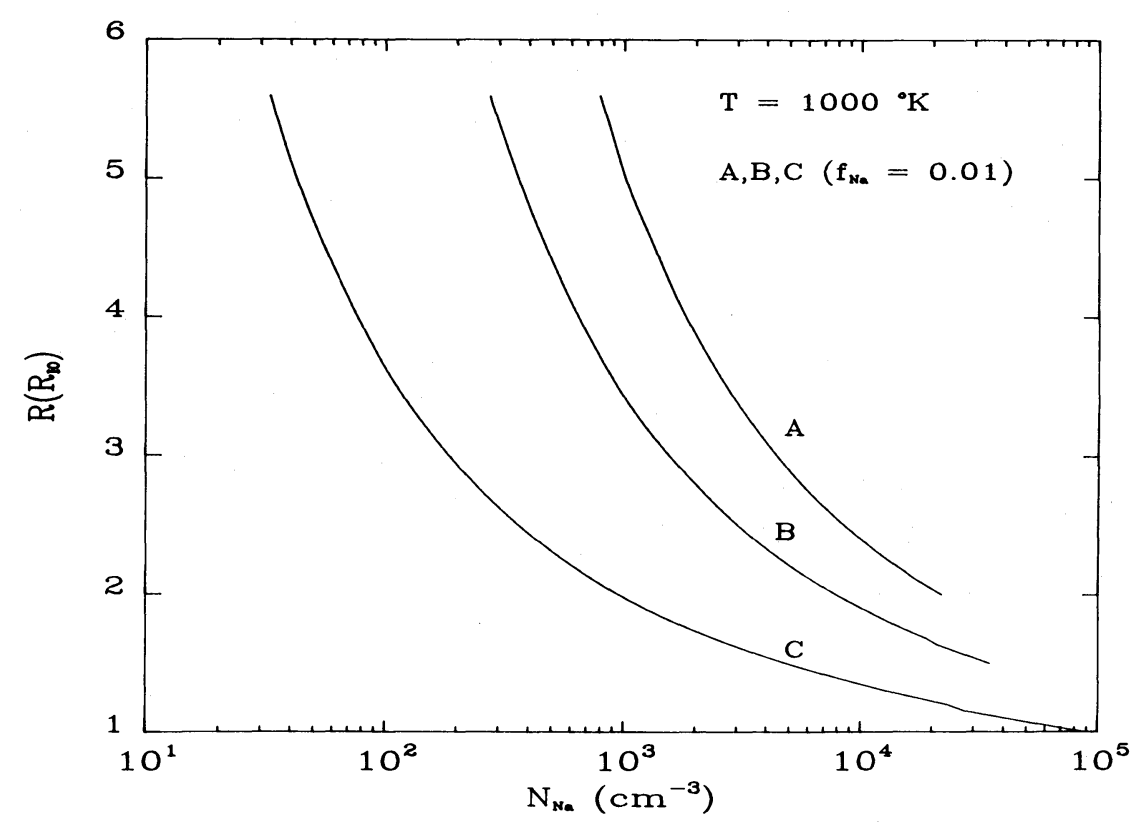

Fig. 2.-Plot of the Na density distribution in Io's corona similar to Fig. 1. The assumed mixing ratio of $\mathrm{Na}$ at the critical level is 0.01 in models A, B, and C.

eters. Figures 1 and 2 show the radial density distributions of $\mathrm{O}$ and $\mathrm{Na}$, respectively. In model $A n_{\mathrm{O}}\left(r_{c}\right)=2.7 \times 10^{6} \mathrm{~cm}^{-3}$ and decreases to $n_{\mathrm{O}}\left(r_{L}\right)=1.9 \times 10^{5} \mathrm{~cm}^{-3}$, where $r_{L}=5.79 R_{\mathrm{Io}}$. The reason for the extremely gradual density decrease with increasing $r$ is the small value of Io's effective gravitational acceleration at these large distances. In an isothermal atmosphere the scale height as defined by $H=k T / m g$ (where $m$ is the mass of an atomic oxygen atom and $g$ is the local gravitational acceleration) increases as $r^{2}$, from a value of $H\left(r_{c}\right)=$ $1150 \mathrm{~km}$ to $H\left(r_{L}\right)=9600 \mathrm{~km}=5.27 R_{\mathrm{Io}}=0.91 r_{L}$.

Changing the location of the critical level substantially affects the coronal density at large distances from Io. In model $\mathrm{B}$ with an assumed $r_{c}=1.5 R_{\mathrm{Io}}, n_{\mathrm{O}}\left(r_{c}\right)$ is larger in case $\mathrm{B}$ than in case $\mathrm{A}$ because of the smaller scale height at $1.5 R_{\mathrm{Io}}$. The effect on the density at $r=r_{L}$ is a decrease from the case $A$ value by a factor of 2. Setting $r_{c}=1.0 R_{\mathrm{Io}}$, i.e., Io's surface, reduces the density at $r=r_{L}$ by another factor of 4.2 over case B. However, it is still worthy of note that even for an exobase located at Io's surface, the density at $r=r_{L}$ is still $\sim 10^{4} \mathrm{~cm}^{-3}$, a value $\sim 10^{2}$ times larger than inferred average values of the neutral density in the plasma torus (Smith and Strobel 1985; T. E. Skinner, 1986, personal communication). The implications of this will be discussed more in $\S \mathrm{IV}$. Cases $\mathrm{D}$ and $\mathrm{E}, T_{c}=1500 \mathrm{~K}$ and $500 \mathrm{~K}$, respectively, illustrate the temperature sensitivity (see Figure 1b).

Sodium's larger mass (and correspondingly smaller scale height) than atomic oxygen produces a greater sensitivity of $n_{\mathrm{Na}}(r)$ to the assumed values of $r_{c}$ and $T_{c}$, as seen in Figure 2. This is important as the distribution of $\mathrm{Na}$ in the corona is tied to the observations of $\mathrm{Na}$ jets and eclipse absorption features mentioned earlier.

If we assume no deflection of torus plasma flow around Io, which is only approximately correct even in the outer regions of the corona (see $\S \mathrm{IV}$ ), an ion's path through the corona is roughly parallel to Io's orbital velocity vector. We ignore the $\sim 11^{\circ}$ tilt of the Jovian dipolar magnetic field to Io's orbital plane. Figures 3 and 4 show the calculated column density of neutral coronal atoms for $\mathrm{O}$ and $\mathrm{Na}$, respectively, along an undeflected ion's path as a function of distance of closest approach of the ion to Io. Adopting a value of $\sigma_{c}=3 \times 10^{-15}$ $\mathrm{cm}^{2}$ for a typical value of the atomic interaction cross section, it is seen that the corona is collisionally "thick" to the incoming ions to a radius of $4.3 R_{\mathrm{Io}}$, for model $\mathrm{A}$. This is significant for two main reasons.

First, in such an extended "thick" exosphere ion-atom and electron-atom interactions occur at distances beyond the critical level. Thus the effective "size" of Io's atmosphere for interaction with the corotating plasma is larger than that determined by the cross sectional size of the collisiondominated region. Second, the efficiency of many escape mechanisms are a function of the gravitational binding energy of the atoms to the satellite. The further from Io that the escape mechanism is operative, the more efficient it is likely to be. For example, both thermal escape and atmospheric sputtering and

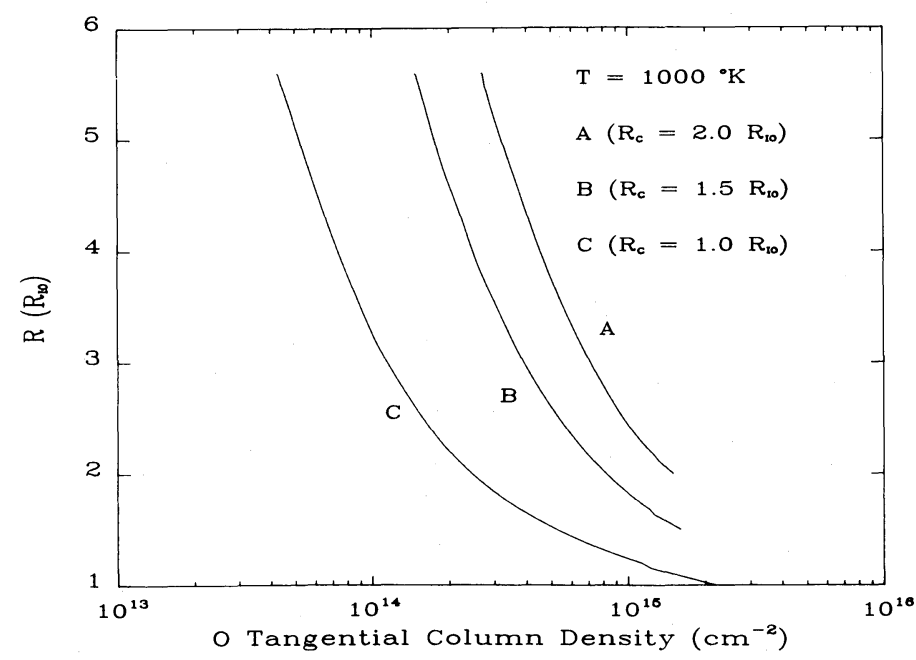

FIG. 3.-The tangential column density of atomic oxygen in Io's corona as a function of distance of closest approach of the tangent path to Io's center. These column densities represent those through which an undeviated torus ion will pass. 


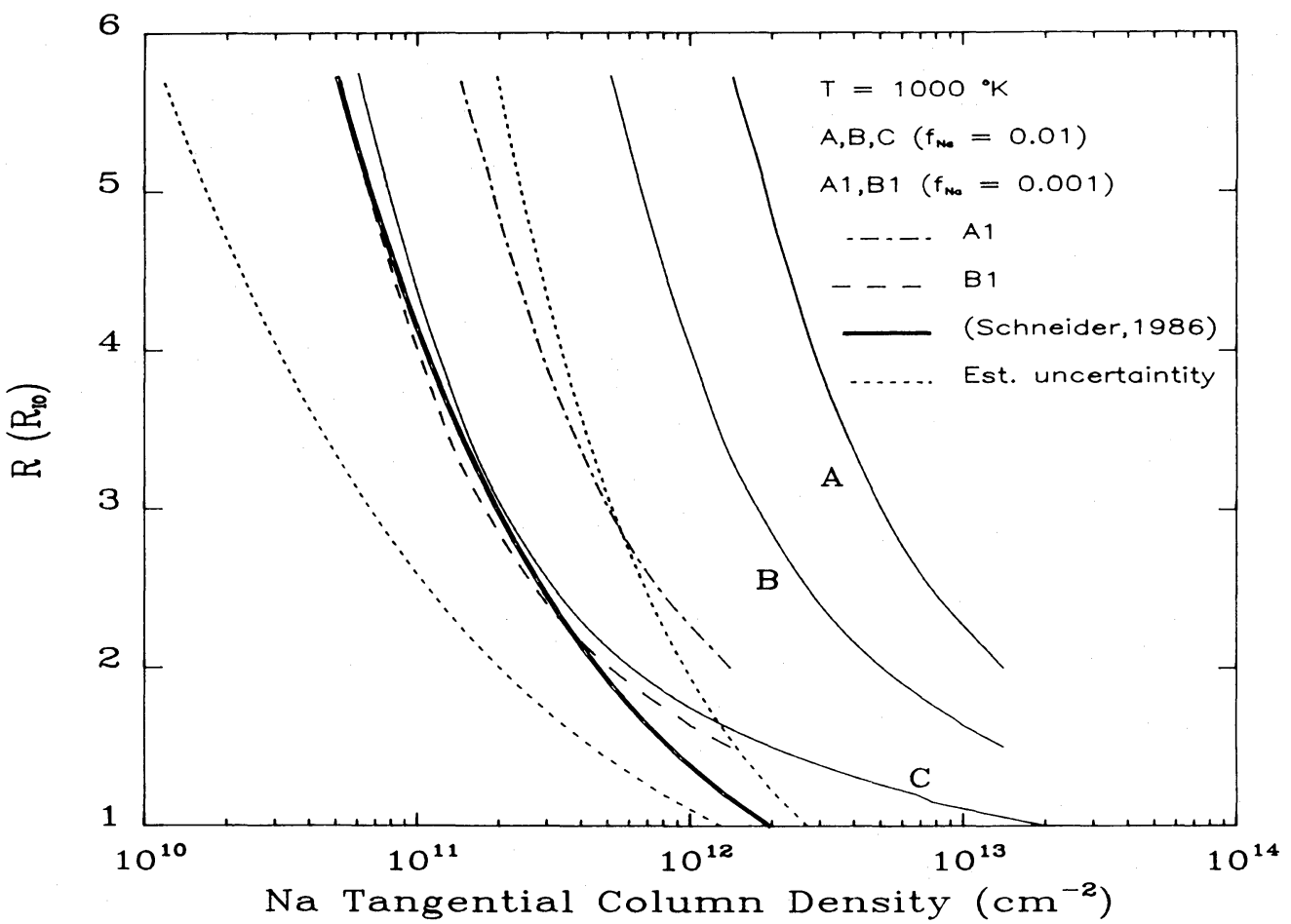

FIG. 4.-The tangential column density of Na plotted as in Fig. 3. The tangential column density of Na models A, B, and C are shown as solid lines. Models A1

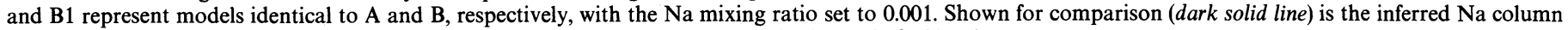
density as deduced by Schneider et al. (1987) along with their estimated uncertainties (short dashed lines).

knock-on processes become more efficient with increasing distance from Io. Escape processes such as charge exchange or ion/electron impact ionization followed by pickup by the corotating Jovian magnetic field can be more effective at removing material from Io's extended exosphere than below the critical level simply because more atoms are "exposed" to corotating plasma.

\section{b) Sulfur-dominated Corona}

We here consider two cases in which $S$ is the dominant constituent at the exobase. The critical level location and temperature are the same as in model $\mathrm{A}$. In case $\mathrm{F}, \mathrm{S}$ has a mixing ratio of 0.5 at the critical level. This has the effect of increasing the $\mathrm{S}$ density at $r=r_{L}$ by a factor of 5.0 over model A. The total number of S atoms "exposed", i.e., above the critical level and not in Io's shadow region, is approximately proportional to the $\mathrm{S}$ mixing ratio at $r=r_{c}$. Atomic oxygen is the dominant constituent throughout most of the corona (except near the critical level where its abundance is about $2 \%$ less than that of S) and a factor of 4.9 more abundant than $\mathrm{S}$ at $r=r_{L}$.

In model $G$ we have fixed $f_{s}\left(r_{c}\right)=0.9$. In this case, the $S$ component is dominant for $r>r_{c}$. The density of $\mathrm{S}$ at $r=r_{L}$ is $3.5 \times 10^{4} \mathrm{~cm}^{-3}$, a factor of 2.0 higher than that of $O$. The total ion path column densities are substantially different in models A and G. For model G the atmosphere is "collisionally thick" to an incoming ion to a radius $r=2.8 r_{\mathrm{Io}}$. The density distributions of $\mathrm{Na}$ and $\mathrm{K}$ in the S-dominated corona are very similar to those calculated for case A. In the next section we investigate the implications of an extended Io corona on atmospheric escape.

\section{ATMOSPHERIC ESCAPE}

The escape of mass from Io's corona can occur via thermal or nonthermal processes. Some of the many possible escape mechanisms that can occur on terrestrial bodies are reviewed by Hunten (1982). Here we briefly review and discuss some of these in the context of interactions between Io's corona and both the plasma torus and Jovian magnetosphere. Each of these processes give specific and distinct velocity signatures for the escaping particles.

\section{a) Escape Processes}

The torus parameters we adopt are those of Johnson and Strobel (1982) appropriate for Io's orbital radius of $5.9 R_{\mathrm{J}}$ :

$$
\begin{aligned}
T_{e} & =5 \mathrm{eV} \\
n_{e} & =1900 \mathrm{~cm}^{-3} \\
n\left(\mathrm{O}^{+}\right) & =600 \mathrm{~cm}^{-3} \\
n\left(\mathrm{~S}^{+}\right) & =165 \mathrm{~cm}^{-3} \\
n\left(\mathrm{O}^{++}\right) & =20 \mathrm{~cm}^{-3} \\
n\left(\mathrm{~S}^{++}\right) & =450 \mathrm{~cm}^{-3} \\
n\left(\mathrm{~S}^{++}\right) & =65 \mathrm{~cm}^{-3}
\end{aligned}
$$

We assume small $\left(<10^{-2}\right)$, torus mixing ratios of $\mathrm{Na}^{+}$and $\mathrm{K}^{+}$ to investigate coronal charge exchange with these species. This requires minor modification of the above parameters for consistency.

i) Thermal Escape

Classical thermal (Jeans) escape is the most well studied and formulated escape process. The escape flux of constituent $i$ at $r=r_{c}$ is given by

$$
\phi_{i}{ }^{T}=n_{i}\left(r_{c}\right) \frac{C_{i}}{\sqrt{2 \pi}}\left(1+\lambda_{c}\right) e^{-\lambda_{c}} .
$$


where

$$
C_{i}=\left(\frac{k T}{m_{i}}\right)^{1 / 2} .
$$

The total escape rate from Io is therefore the integration of the above escape flux over the surface formed by the critical level. We assume that thermal escape of each constituent is independent of that of the other constituents. Typically only the highest energy atoms, those in the high-energy tail of the Maxwell-Boltzmann distribution, will escape. However, most of the excess energy (the difference between the atoms' kinetic energy and the energy needed to escape) is converted to potential energy as the atom moves out of the gravitational well of Io. Thus the average kinetic energy of the "escaped" atoms is comparable (say within 33\%) to the average kinetic energy of the atoms at the critical level.

\section{ii) Atmospheric Sputtering and Knock-on Processes}

The erosion of planetary and satellite atmospheres by impacting energetic atomic particles with applications to Mars and Ganymede has been investigated by Haff and Watson (1979). A similar study with applications to Io was carried out by Watson (1981) and Haff, Watson and Yung (1981). Analytic expression for the sputter-erosion yield were presented by Haff and Watson (1981) which give the sputtering yield as a function of target density, binding energy of the atom to the target, and nuclear stopping power. The nuclear stopping power is in turn dependent upon the energy of the bombarding particle and the relative masses and nuclear charges of the bombarding and target particles. Using these expressions presented in Haff and Watson (1981), we have calculated the sputtering yield for O, S, $\mathrm{Na}$, and $\mathrm{K}$ torus ions impacting Io's atmosphere. Figure 5 shows the calculated variation of sputtering yield for corotating ions impacting the critical level as a function of critical level radius. This type of sputtering is generally referred to as classical collision cascade sputtering and is the backward recoil of particles due to multiple collisions initiated by a high-energy ion impact. This process requires a collisionally "thick" target. A typical "hard" collision cross section is a few times $10^{-16}$ $\mathrm{cm}^{2}$. This rate of escape of constituent $i$ due to impacting ion $j$ is approximately

$$
\phi_{i}^{S}=\pi r_{c}^{2} F_{j} Y_{j i}\left(r_{c}\right) .
$$

where $F_{j}=n_{j} \Delta u$ is the flux of ion $j, n_{j}$ is the torus number density, $\Delta u$ is the relative velocity between Io and the incoming ions, and $Y_{j i}$ is the yield of atom $i$ from impacting ion $j$. For sputtering from a target containing a mixture of atoms by a plasma containing several distinct ion species, the appropriate weighted average must be taken to obtain the individual atomic sputtering rates.

The physics of ion-atom collisions in the context of interaction between the plasma torus and the diffuse neutral atomic clouds which are not gravitationally bound to Io has been discussed briefly by Brown, Pilcher, and Strobel (1983). Knock-on is the process whereby a target atom obtains forward kinetic energy in an ion/atom collision. "Soft" collisions result from the impact of an ion with an atom with a high polarizability, like $\mathrm{Na}$ and $\mathrm{K}$, and typically have larger collision cross sections than "hard" collisions. Table 3 is a list of knock-on collisions possible in Io's atomic corona. We adopt $\sigma_{H}=3 \times 10^{-16} \mathrm{~cm}^{2}$ for the "hard" momentum transfer cross section, and use Brown et al. (1983) to calculate the "soft" collision cross sections, which are a function of the target atoms polarizability.

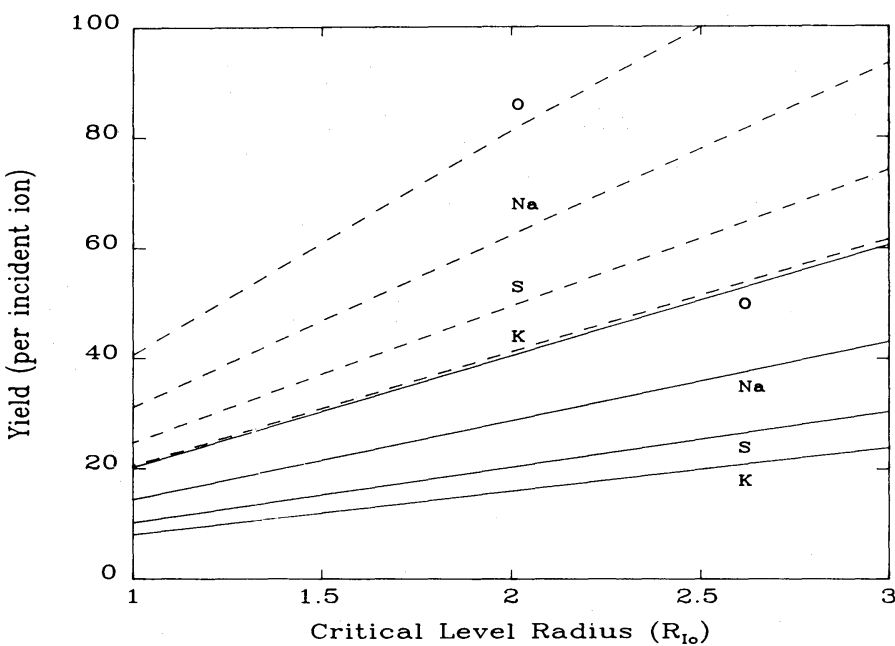

FiG. 5.-Plot of atmospheric sputtering yield (atoms ejected per incident ion) for incident $\mathrm{O}+($ solid lines $)$ and $\mathrm{S}+($ dashed lines $)$ on pure atomic $(\mathrm{O}, \mathrm{S}$, $\mathrm{Na}$, and $\mathrm{K}$ ) atmospheres on Io as a function of the critical level radius

The escape velocity is $1.81 \mathrm{~km} \mathrm{~s}^{-1}(0.27 \mathrm{eV}$ and $0.67 \mathrm{eV}$ for $\mathrm{O}, \mathrm{Na}$, respectively) at $r=2 R_{\mathrm{Io}}$. Corotating oxygen and sulfur ions at Io's orbit have kinetic energies relative to Io of $280 \mathrm{eV}$ and $560 \mathrm{eV}$, respectively. Considering the knock-on processes in Table 3 , the maximum kinetic energy transferrable is in every case greater than $82 \%$ of the ion's initial kinetic energy. If all energy transfers are equally probable, then one expects the vast majority of coronal atoms undergoing one of the reactions to Table 3 to obtain more than Io's escape velocity, certainly more than the atoms' mean thermal velocity of $\sim 0.5 \mathrm{~km} \mathrm{~s}^{-1}$. If on average $\sim \frac{1}{2}$ of the ion's initial kinetic energy is transferred to the neutral atom, then the escaping atoms will have sufficient energy so that further collisions with coronal atoms will not prevent escape.

We assume that knock-on occurs in that region where the plasma impacts the corona above the target disk which has a radius of $r_{p}$, centered on Io. The total escape rate for constituent $i$ is thus given by the integral over the collision volume of the relevant cross section for ion $j$ impacting neutral $i$, multiplied by the density of constituent $i$ and the flux of ion $j$, i.e.,

$$
\phi_{i}=\int_{V} \sigma_{i j} n_{i} F_{j} d v .
$$

We assume that the total flux given by $\phi_{i}$ escapes from Io. In fact this escape rate overestimates the actual escape rate if the

TABLE 3

\begin{tabular}{|c|c|c|}
\hline Model & Reaction & $\sigma\left(\mathrm{cm}^{2}\right)$ \\
\hline & $\mathrm{O}^{+}+\mathrm{O} \rightarrow \mathrm{O}^{+}+\mathrm{O}^{*}$ & $3.0 \times 10^{-16}$ \\
\hline $\mathrm{K} 2$. & $\mathrm{O}^{+}+\mathrm{S} \rightarrow \mathrm{O}^{+}+\mathrm{S}^{*}$ & $3.0 \times 10^{-16}$ \\
\hline $\mathrm{K} 3$. & $\mathrm{O}^{+}+\mathrm{Na} \rightarrow \mathrm{O}^{+}+\mathrm{Na}^{*}$ & $7.4 \times 10^{-16 a}$ \\
\hline $\mathrm{K} 4$.. & $\mathrm{O}^{+}+\mathrm{K} \rightarrow \mathrm{O}^{+}+\mathrm{K}^{*}$ & $8.0 \times 10^{-16 \mathrm{a}}$ \\
\hline K5.. & $\mathrm{S}^{+}+\mathrm{O} \rightarrow \mathrm{S}^{+}+\mathrm{O}^{*}$ & $3.0 \times 10^{-16}$ \\
\hline K6.. & $\mathrm{S}^{+}+\mathrm{S} \rightarrow \mathrm{S}^{+}+\mathrm{S}^{*}$ & $3.0 \times 10^{-16}$ \\
\hline K7.. & $\mathrm{S}^{+}+\mathrm{Na} \rightarrow \mathrm{S}^{+}+\mathrm{Na}^{*}$ & $6.3 \times 10^{-16 \mathrm{a}}$ \\
\hline $\mathrm{K} 8$. & $\mathrm{S}^{+}+\mathrm{K} \rightarrow \mathrm{S}^{+}+\mathrm{K}^{*}$ & $1.3 \times 10^{-15 \mathrm{a}}$ \\
\hline
\end{tabular}

Momentum Transfer Reactions and Cross Sections Adopted for Use in KNOCK-ON Escape Rate Calculations

a Collisions involving $\mathrm{Na}$ and $\mathrm{K}$ are considered "soft" reactions (see text). 
volume over which the integration is taken is for impact parameter $r_{p} \geq r_{c}$, i.e., above the critical level, since some of the fast atoms are thermalized before escaping. Therefore in our calculation of the knock-on escape rate we assume that the volume used in the integration for equation (9) is that where the corona is collisionally thin to escaping particles, e.g., for model A this would be $r_{p} \geq 4.3 R_{\mathrm{Io}}$.

\section{iii) Charge Exchange}

Torus ions undergo charge exchange with coronal atoms and produce a high-velocity neutral and a newly created ion which is then accelerated to corotation velocity. Table 4 contains several charge exchange reactions which are probably important in the corona (taken from Johnson and Strobel 1982). Here we have not attempted to be comprehensive in the listing; we have chosen those reactions which may lead to high-velocity "jets" of neutral atoms. The escape flux due to charge exchange reactions is calculated in the same way as for the knock-on escape flux (eq. [9]) with the appropriate cross sections.

\section{iv) Electron Impact Ionization}

Electron impact ionization coefficients for $\mathrm{Na}$ and $\mathrm{K}$ have been calculated by Brown et al. and those for $\mathrm{O}$ and $\mathrm{S}$ by Smith and Strobel (1985). The ionization coefficients we use are

$$
\begin{aligned}
I(\mathrm{O}) & =7.9 \times 10^{-9} \mathrm{~cm}^{3} \mathrm{~s}^{-1} \\
I(\mathrm{~S}) & =1.8 \times 10^{-8} \mathrm{~cm}^{3} \mathrm{~s}^{-1} \\
I(\mathrm{Na}) & =5.8 \times 10^{-8} \mathrm{~cm}^{3} \mathrm{~s}^{-1} \\
I(\mathrm{~K}) & =8.0 \times 10^{-8} \mathrm{~cm}^{3} \mathrm{~s}^{-1}
\end{aligned}
$$

and the ionization rate is given by

$$
J_{i}=I_{i} n_{e} n \sqrt{T_{e}} \exp \left(\frac{-I_{p}}{T_{e}}\right),
$$

where $I_{p}$ is the ionization potential, $T_{e}$ is the electron temperature $(\mathrm{eV}), n_{e}$ the electron density, and $n$ the neutral density. The ionization rate is a sensitive function of electron temperature. The actual electron impact ionization rate in the atomic corona surrounding Io will strongly depend upon how the torus plasma properties are changed by the interaction. We assume that electron impact ionization occurs only above the critical level, and that the physical state of the plasma (e.g., $n_{e}$, $T_{e}$ ) is constant in this region. Substantial cooling of the plasma in the interaction with the neutral corona is likely to occur in this region (see Ballester et al. 1987), and since we are assuming a constant electron density our estimates of the total escape rate due to this process can be considered an upper limit. The

TABLE 4

Charge Exchange Reactions

\begin{tabular}{ccc}
\hline \hline Model & Reaction & $\sigma\left(\mathrm{cm}^{2}\right)$ \\
\hline $\mathrm{C} 1 \ldots \ldots \ldots$ & $\mathrm{O}^{+}+\mathrm{O} \rightarrow \mathrm{O}^{*}+\mathrm{O}^{+}$ & $2.2 \times 10^{-15 \mathrm{a}}$ \\
$\mathrm{C} 2 \ldots \ldots \ldots$. & $\mathrm{O}^{+}+\mathrm{S} \rightarrow \mathrm{O}^{*}+\mathrm{S}^{+}$ & $1.6 \times 10^{-15 \mathrm{a}}$ \\
$\mathrm{C} 3 \ldots \ldots \ldots$ & $\mathrm{S}^{+}+\mathrm{O} \rightarrow \mathrm{S}^{*}+\mathrm{O}^{+}$ & $1.0 \times 10^{-17 \mathrm{a}}$ \\
$\mathrm{C} 4 \ldots \ldots \ldots$ & $\mathrm{S}^{+}+\mathrm{S} \rightarrow \mathrm{S}^{*}+\mathrm{S}^{+}$ & $2.9 \times 10^{-15 \mathrm{a}}$ \\
$\mathrm{C} 5 \ldots \ldots \ldots$ & $\mathrm{Na}^{+}+\mathrm{Na} \rightarrow \mathrm{Na}^{*}+\mathrm{Na}^{+}$ & $1.5 \times 10^{-14 \mathrm{~b}}$ \\
$\mathrm{C} 6 \ldots \ldots \ldots$ & $\mathrm{K}^{+}+\mathrm{K} \rightarrow \mathrm{K}^{*}+\mathrm{K}^{+}$ & $2.0 \times 10^{-14 \mathrm{~b}}$ \\
\hline
\end{tabular}

a Taken from Johnson and Strobel 1982, $\sigma_{\mathrm{C} 3}$ is an upper limit.

${ }^{b}$ From Smirnov 1965.
TABLE 5

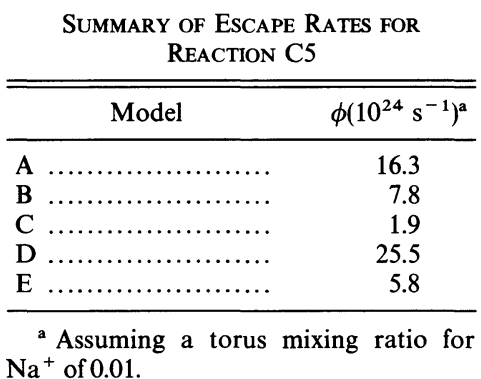

total escape rate is thus given by

$$
\phi_{i}=\int_{V} J_{i} d v,
$$

The volume of integration used here is for that impact parameter $r_{p} \geq r_{c}$. We are thus assuming that plasma flow reaches to the critical level and ions which are created are then swept into the magnetosphere. Collisions between these newly created ions and other coronal atoms will change the velocity of the new ion, but we assume the corotation electric field reenergizes the ion between collisions.

\section{b) Neutral Sodium "Jets"}

We first consider the generation of the observed high velocity "jets." The most obvious possibility to investigate is the resonant charge exchange reaction $\mathrm{C5}$ which has a cross section of $1.5 \times 10^{-14} \mathrm{~cm}^{2}$. From Figure 4 we see that for the assumed model parameters, the $\mathrm{Na}$ coronal component is collisionally thin to reaction $\mathrm{C}$. Once a high-velocity $\mathrm{Na}$ is produced by $\mathrm{C} 5$ it will likely escape from Io's atmosphere without charge exchanging back to an ion. However, in view of the large ion path column densities of neutral species in the corona, the fast $\mathrm{Na}$ will likely experience momentum transfer collisions on its trajectory subsequent to the charge exchange reaction. For a minor coronal constituent with $f_{i}\left(r_{c}\right) \ll 1$ the ion path column density is proportional to $f_{i}\left(r_{c}\right)$.

Assuming no deviation of plasma flow and insignificant changes in plasma properties through the corona, the escape rate due to reaction $\mathrm{C} 5$ is approximately

$$
\phi_{\mathrm{Na}}{ }^{\mathrm{C} 5}=\sigma_{\mathrm{C} 5} N_{\mathrm{Na}} F_{\mathrm{Na}^{+}},
$$

where $N_{\mathrm{Na}}$ is the total number of $\mathrm{Na}$ atoms in Io's corona in the region that is collisionally thin to incoming ions, i.e., for model $\mathrm{A}$ this is for impact parameter $r_{p} \geq 4.3 R_{\mathrm{Io}}$. $F_{\mathrm{Na}}$ is the flux $\left(\mathrm{cm}^{-2} \mathrm{~s}^{-1}\right)$ of $\mathrm{Na}^{+}$impacting Io's corona. The density of $\mathrm{Na}^{+}$in the plasma torus is poorly constrained and we use $1 \%$ in the following calculations. The escape rates can therefore be scaled to the correct value as information on the mixing ratio of $\mathrm{Na}$ ions in the torus becomes available.

Table 5 is a summary of models which produce high-velocity $\mathrm{Na}$ ejection by reaction $\mathrm{C} 5$. As mentioned in the Introduction, one of the major observed characteristics of the $\mathrm{Na}$ "jet" near Io is that the cross sectional intensity profile has a FWHM of 2-3 Io diameters. The shape of the cross sectional intensity profile of the fast $\mathrm{Na}$ jet contains information on the distribution of $\mathrm{Na}$ in Io's atmosphere. The Earth-based observations of the $\mathrm{Na}$ jet have a spatial resolution of approximately 1 " or one Io diameter. This coarse spatial resolution does not permit one to map the Na coronal distribution in detail. 


\section{c) Total Escape Rates}

Here we consider in detail the escape of atoms from Io's atmosphere by means of the processes discussed in $\S$ IV. Photochemical models of Io's atmosphere which reproduce the major characteristics of Io's observed daytime ionosphere imply critical level conditions best matched by models $A$ and B. Model $\mathrm{C}$ is incompatible with ionosphere models as the atmosphere is too thin to provide an optically thick target to solar UV radiation or energetic electrons. The atmosphere must be optically thick so that a peak in the electron density will form (as in the classical Chapman theory). Models D and $E$ are variations on model $A$ and in terms of atmospheric escape are not significantly distinct from A (see Table 8). Below we will consider in detail the atomic escape rates calculated for model B. The total escape rates for each model are listed in Table 8 .

\section{i) Model B-Oxygen-dominated Corona}

We consider model $B$ as the prototype for discussion of escape from an atomic oxygen dominated corona. Table 6 contains the results of the escape calculations for each mechanism and each individual constituent. Atomic oxygen has the largest escape rate. The $O$ mass-loss rate is $3 \times 10^{3} \mathrm{~kg} \mathrm{~s}^{-1}$, about a factor of 5 higher than the upper limit derived by Dessler (1980), and a factor of 7 higher than the theoretically calculated supply rate for the oxygen torus (Smith and Strobel 1985). In $\S \mathrm{V}$ we will discuss the model assumptions which imply such large escape rates.

The escape mechanism with the largest rate for all constituents is atmospheric sputtering, which contributes $69 \%, 59 \%$, $89 \%$, and $82 \%$ of the total escape rates of $\mathrm{O}, \mathrm{Na}, \mathrm{S}$, and $\mathrm{K}$, respectively. Electron impact ionization is second in importance for total escape rates, again for all constituents. For the two least massive constituents, $\mathrm{O}$ and $\mathrm{Na}$, thermal escape dominates over the charge exchange reactions in Table 4. However for $\mathrm{S}$ escape, charge exchange is about 4 times more efficient. This is due primarily to reaction $\mathrm{C} 2$ which has a reaction cross section comparable to typical molecular collision cross sections. For $\mathrm{K}$, thermal escape and reaction $\mathrm{C6}$ have comparable rates. Momentum transfer reactions have the lowest escape rates for $\mathrm{O}$, but are comparable to thermal escape and charge exchange for $\mathrm{Na}$. The $\mathrm{O}$ escape rate is 13 times that of $S$, about half the relative $O / S$ rate needed for the "oxygen torus" of Smith and Strobel (1985). In this model (B) the main determinant of the relative magnitude of the escape rates for $\mathrm{O}$ and $\mathrm{S}$ is simply the $f_{i}\left(r_{c}\right)$.

\section{ii) Model G-Sulfur-dominated Corona}

In model $B$ oxygen has a mixing ratio of about 0.9 at the critical level. In model $G$ we set the mixing ratio of $S$ at 0.9 to investigate the escape from an atmosphere that has been injected with large amounts of sulfur.

The mass escape rate from model $G$ is almost 4 times as large as model $\mathrm{B}$. This puts the mass injection rate more than an order of magnitude larger than the limit by Dessler (1980). On the other hand, impulsive injection of $S$, whether by $S$ volcanoes or evaporation from sulfur lakes, is expected to last a short time (hours to possibly a day). The Dessler limit should therefore be considered a time-averaged upper limit (i.e., over a few weeks or longer). But sulfur ejection should have substantial short-term effects on mass loading of the torus.

In terms of escape processes and rates (see Table 7) atmospheric sputtering is again the dominant process, contributing $60 \%, 61 \%, 87 \%$, and $78 \%$ for $\mathrm{O}, \mathrm{Na}, \mathrm{S}$, and $\mathrm{K}$, respectively. For $\mathrm{S}$, atmospheric sputtering is followed by electron impact ionization in importance. But for $\mathrm{O}$, thermal escape, electron impact ionization, and charge exchange ( $\mathrm{C1}$ and $\mathrm{C} 3)$ are all of comparable importance. The relative escape rate of $\mathrm{S} / \mathrm{O}$ is about 8, while the "sulfur torus" of Smith and Strobel (1985) requires a relative rate of 6.5 .

\section{DISCUSSION}

The key assumption in the models of Io's corona presented

TABLE 6

Atomic Escape Rates for Model B (atoms s ${ }^{-1}$ )

\begin{tabular}{|c|c|c|c|c|}
\hline Parameter & $\mathrm{O}$ & $\mathrm{Na}$ & $\mathrm{S}$ & $\mathrm{K}$ \\
\hline Thermal Escape . & $8.30 \times 10^{27}$ & $1.52 \times 10^{25}$ & $1.57 \times 10^{25}$ & $2.65 \times 10^{22}$ \\
\hline Sputtering: $\ldots \ldots \ldots \ldots \ldots \ldots \ldots$ & $7.99 \times 10^{28}$ & $8.07 \times 10^{26}$ & $8.08 \times 10^{27}$ & $8.07 \times 10^{25}$ \\
\hline \multicolumn{5}{|l|}{ Knock-on: } \\
\hline $\mathrm{K} 1 \ldots \ldots$ & $1.02 \times 10^{27}$ & $\cdots$ & & \\
\hline $\mathrm{K} 2$ $\ldots \ldots \ldots \ldots \ldots \ldots \ldots \ldots \ldots \ldots$ & $\ldots$ & $\ldots$ & $2.05 \times 10^{25}$ & \\
\hline K3 $\ldots \ldots$ & $\ldots$ & $1.22 \times 10^{25}$ & $\ldots$ & \\
\hline n............ & $\ldots$ & $\ldots$ & $\ldots$ & $3.21 \times 10^{23}$ \\
\hline n............... & $2.80 \times 10^{26}$ & $\ldots$ & & \\
\hline $\mathrm{K} 6 \ldots \ldots$ & $\ldots$ & $\ldots$ & $5.62 \times 10^{24}$ & \\
\hline K7 $\ldots \ldots \ldots \ldots \ldots \ldots \ldots \ldots \ldots$ & $\ldots$ & $2.85 \times 10^{24}$ & $\ldots$ & \\
\hline K8 $\ldots \ldots \ldots \ldots \ldots \ldots \ldots \ldots$ & $\ldots$ & $\ldots$ & $\ldots$ & $1.43 \times 10^{23}$ \\
\hline Total(KO): $\ldots \ldots \ldots \ldots \ldots \ldots \ldots$ & $1.30 \times 10^{27}$ & $1.50 \times 10^{25}$ & $2.61 \times 10^{25}$ & $4.64 \times 10^{23}$ \\
\hline Electron Impact Ionization: .... & $1.87 \times 10^{28}$ & $5.27 \times 10^{26}$ & $9.06 \times 10^{26}$ & $1.77 \times 10^{25}$ \\
\hline \multicolumn{5}{|l|}{ Charge Exchange: } \\
\hline $\mathrm{C} 1 \ldots \ldots \ldots \ldots$ & $7.46 \times 10^{27}$ & & & \\
\hline $\mathrm{C} 2 \ldots \ldots \ldots \ldots \ldots \ldots \ldots \ldots$ & $\ldots$ & $\ldots$ & $1.09 \times 10^{26}$ & \\
\hline $\mathrm{C} 3 \ldots \ldots \ldots$ & $9.33 \times 10^{24}$ & & & \\
\hline 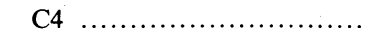 & $\ldots$ & $\cdots$ & $5.45 \times 10^{25}$ & \\
\hline $\mathrm{C} 5$.......................... & $\ldots$ & $7.80 \times 10^{24}$ & & \\
\hline $\mathrm{C} 6$ (n..................... & $\ldots$ & $\ldots$ & & $2.54 \times 10^{22}$ \\
\hline Total(CE): $\ldots \ldots \ldots \ldots \ldots \ldots \ldots$ & $7.57 \times 10^{27}$ & $7.80 \times 10^{24}$ & $6.38 \times 10^{25}$ & $2.54 \times 10^{23}$ \\
\hline Total (w/out sputtering): ....... & $3.61 \times 10^{28}$ & $5.63 \times 10^{26}$ & $1.01 \times 10^{27}$ & $1.82 \times 10^{25}$ \\
\hline Total (all mechanisms): ........ & $1.16 \times 10^{29}$ & $1.37 \times 10^{27}$ & $9.09 \times 10^{27}$ & $9.90 \times 10^{25}$ \\
\hline
\end{tabular}


TABLE 7

\begin{tabular}{|c|c|c|c|c|}
\hline Parameter & $\mathrm{O}$ & $\mathrm{Na}$ & $\mathrm{S}$ & $\mathbf{K}$ \\
\hline Thermal Escape: . & $2.04 \times 10^{27}$ & $6.61 \times 10^{25}$ & $1.12 \times 10^{25}$ & $3.33 \times 10^{23}$ \\
\hline Sputtering: $\ldots \ldots \ldots \ldots \ldots \ldots \ldots \ldots \ldots$ & $1.70 \times 10^{28}$ & $1.91 \times 10^{27}$ & $1.72 \times 10^{29}$ & $1.91 \times 10^{26}$ \\
\hline \multicolumn{5}{|l|}{ Knock-on: } \\
\hline $\mathrm{K} 1 \ldots \ldots$ & $1.41 \times 10^{26}$ & & & \\
\hline $\mathrm{K} 2 \ldots \ldots$ & .. & & $4.20 \times 10^{26}$ & \\
\hline K3 $\ldots .$. & $\ldots$ & $2.54 \times 10^{25}$ & & \\
\hline $\mathrm{K} 4 \ldots \ldots$. & $\ldots$ & $\ldots$ & $\ldots$ & $9.48 \times 10^{23}$ \\
\hline$\ldots \ldots \ldots \ldots \ldots \ldots \ldots \ldots \ldots$ & $4.61 \times 10^{25}$ & & & \\
\hline 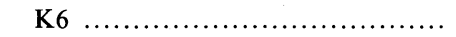 & $\ldots$ & & $1.38 \times 10^{26}$ & \\
\hline $\mathrm{K} 7 \ldots \ldots \ldots \ldots$ & $\ldots$ & $5.96 \times 10^{24}$ & & \\
\hline $\mathrm{K} 8 \ldots \ldots \ldots$ & $\ldots$ & & & $4.24 \times 10^{23}$ \\
\hline Total $(\mathrm{KO}):{ }_{1} \ldots \ldots \ldots \ldots \ldots \ldots \ldots \ldots$ & $1.87 \times 10^{26}$ & $3.14 \times 10^{25}$ & $5.58 \times 10^{26}$ & $1.37 \times 10^{24}$ \\
\hline Electron Impact Ionization: ........... & $2.60 \times 10^{27}$ & $1.10 \times 10^{27}$ & $2.22 \times 10^{28}$ & $5.24 \times 10^{25}$ \\
\hline \multicolumn{5}{|l|}{ Charge Exchange: } \\
\hline $\mathrm{C} 1 \ldots \ldots \ldots \ldots$ & $1.03 \times 10^{27}$ & $\ldots$ & & \\
\hline $\mathrm{C} 2$................... & & & $2.68 \times 10^{27}$ & \\
\hline 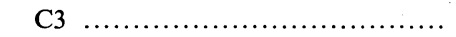 & $1.29 \times 10^{24}$ & $\ldots$ & & \\
\hline 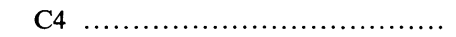 & & & $1.33 \times 10^{24}$ & \\
\hline $\mathrm{C} 5 \quad \ldots \ldots \ldots \ldots \ldots \ldots \ldots$ & & $1.63 \times 10^{25}$ & & \\
\hline $\mathrm{C} 6 \quad \ldots \ldots \ldots \ldots \ldots$ & & & & $7.50 \times 10^{22}$ \\
\hline Total(CE): $\ldots \ldots \ldots \ldots \ldots \ldots \ldots \ldots$ & $3.71 \times 10^{27}$ & $1.63 \times 10^{25}$ & $1.34 \times 10^{27}$ & $7.50 \times 10^{22}$ \\
\hline Total (w/out Sputtering): ............ & $8.50 \times 10^{27}$ & $1.22 \times 10^{27}$ & $2.50 \times 10^{28}$ & $5.50 \times 10^{25}$ \\
\hline Total (All Mechanisms): ............. & $2.55 \times 10^{28}$ & $3.13 \times 10^{27}$ & $1.97 \times 10^{29}$ & $2.46 \times 10^{26}$ \\
\hline
\end{tabular}

in $\S$ III is that only thermal processes determine the radial density structure. If that assumption is correct, Io's corona is extremely extended, and in fact may be collisionally thick to corotating torus ions for an impact parameter $\dot{r}_{p} \leq 4 R_{\mathrm{Io}}$. Such an extensive corona has important implications for the interaction between Io's atmosphere and the Jovian magnetosphere. In fact, the escape rates calculated for model B are larger than the inferred neutral and plasma clouds supply rates by a factor of between 3 and 10, depending upon constituents. Here we examine some of the assumptions made in the preceding calculations. First we consider the possible modification of Io's coronal structure due to the effect of having large thermal and nonthermal escape rates. Second we discuss how the nonthermal escape mechanisms themselves may become less efficient because of the strong torus/atmosphere interaction. Finally we consider observational consequences of these models.

\section{a) Reexamination of Model Assumptions}

The model of Io's exospheric corona constructed in § II assumed that interaction between the torus plasma and the corona produced an insignificant perturbation on the structure of the corona. In view of the large nonthermal escape rates calculated in $\S \mathrm{IV}$, this assumption needs to be reexamined.

The critical level parameters for model $B$ are as follows:

$$
\begin{aligned}
r_{c} & =1.5 R_{\mathrm{Io}} \\
T & =1000 \mathrm{~K}
\end{aligned}
$$

and for atomic oxygen:

$$
\begin{aligned}
\lambda\left(r_{c}\right) & =4.23 \\
H\left(r_{c}\right) & =650 \mathrm{~km} \\
n\left(r_{c}\right) & =4.13 \times 10^{6} \mathrm{~cm}^{-3} \\
U_{s} & =\left(\frac{k T}{m}\right)^{1 / 2}=7.2 \times 10^{4} \mathrm{~cm} \mathrm{~s}^{-1}
\end{aligned}
$$

The population of thermally escaping atoms implies an average radial flow velocity at $r=r_{c}$ of

$$
U_{\mathrm{av}}\left(r_{c}\right)=\frac{\phi_{T}}{n\left(r_{c}\right)}
$$

which for $\mathrm{O}$ is $U_{\mathrm{av}}=2.2 \times 10^{3} \mathrm{~cm} \mathrm{~s}^{-1}$, approximately $3 \%$ of the isothermal sound speed $U_{s}$. The thermal escape flux is about a factor of $\sim 9$ less than the escape flux due to atmospheric sputtering. The total escape flux implies a radial flow velocity of $U_{\text {flow }}=0.42 U_{s}$ at the critical level, assuming $n\left(r_{c}\right)$ is unaffected by such a large (but still subsonic) flow velocity.

It is clear that the velocity distributions of atoms at $r=r_{c}$ is not Maxwellian. The atoms escaping due to sputtering have velocities greater than the local escape velocity $U_{\text {esc }}=2.1 \mathrm{~km}$ $\mathrm{s}^{-1}$, and this component is superposed upon the thermal component. The effect of large escape rates is to decrease the density below that expected for an atmosphere obeying the barometric law (eq. [2] with $\zeta=1$ ). The magnitude of this effect is difficult to quantify for several reasons. First the atoms are sputtered from a fairly thick radial region about the critical level, the radial region probably having a thickness of order $H$. Second, accurate solution of equation (1) requires a knowledge of $n(r)$ for $r \gg r_{c}$ where a major component of the corona may indeed be the sputtered component. The structure of the corona determines how and where sputtering occurs. A fairly complicated feedback mechanism must therefore be examined in order to self-consistently calculate $n(r)$ in the corona. To some extent the critical level density will be depressed due to the rapid escape rate as we show below. Also, the critical level temperature will be modified by the nonthermal interaction, assuming that significant nonthermal processes occur at the critical level. The energy transfer between the torus and the atmosphere and the effect this has on the structure of Io's upper atmosphere has yet to be quantified.

The magnitude of the effect that a large radial flow velocity has on the density profile for $r<r_{c}$ may be estimated as follows. The momentum equation for a radially flowing atmo- 
sphere is given approximately by

$$
n m u \frac{d u}{d r}=-\frac{d P}{d r}-n m \frac{G M}{r^{2}} .
$$

Assuming $P=n k T, T=$ constant, this equation may be integrated between $r_{o}$ and $r$ to give

$$
n(r)=n\left(r_{o}\right) e^{-\left(\lambda_{0}-\lambda\right)-\left(\psi-\psi_{o}\right)},
$$

where

$$
\psi=\frac{1}{2}\left(\frac{u}{u_{s}}\right)^{2}
$$

Equation (15) shows that for a radial flow velocity increasing with increasing $r$ (and $\psi>\psi_{o}$ ), the density profile decreases more rapidly than for the no flow case $\left(\psi=\psi_{o}=0\right)$. To estimate the magnitude of this effect for Io's atmosphere we assume

$$
\phi_{T}=4 \pi r^{2} n u=\text { constant }
$$

for $r_{\mathrm{Io}}<r<r_{c}$. Assuming $\psi_{o}=0$, the maximum correction factor to the density distribution at $r=r_{c}$ is $e^{-\psi_{c}}=0.92$, i.e., an $8 \%$ difference between the flowing solution and the barometric solution. Thus the rapid flow at $r=r_{c}$ has only minor significance (for case B). A much more rapid flow, i.e., $u \sim u_{s}$ at $r=r_{c}$ is needed to significantly perturb the density distribution, but even for $u=u_{s}$ this would produce a correction of only about $35 \%$.

For $r>r_{c}$, one must consider the fact that the thermal corona is losing mass. Electron impact ionization "instantaneously" removes atoms from the corona. Charge exchange also removes coronal atoms and generates highvelocity neutral atoms. These and other processes deplete the population of coronal atoms and hence perturb the density structure for $r>r_{c}$. Here we make a crude model to estimate the magnitude of this perturbation.

The total population of atoms near the critical level has an average radial bulk flow velocity of $U_{\text {flow }}=0.42 \mathrm{~km} \mathrm{~s}^{-1}$. The escaping thermal atoms have an average radial velocity slightly greater than the escape velocity $U_{\text {esc }}=2.1 \mathrm{~km} \mathrm{~s}^{-1}$. The other populations of escaping particles have even larger average velocities. Sputtering, for instance, may produce a splash of atoms with a mean velocity a few times $U_{\text {esc }}$. Charge exchange produces a dispersed jet of atoms with velocities up to and greater than the corotation velocity of $57.3 \mathrm{~km} \mathrm{~s}^{-1}$. Because of the large escape rates the atmosphere/corona is continually expanding with a mean velocity which is a weighted average of the above velocities. The average expansion velocity is difficult to quantify so below we consider several possibilities.

We consider the corona to be expanding as a fluid with a radial flow velocity $u(r)$. The local loss of coronal atoms by nonthermal processes we parameterize in terms of a loss rate coefficient $\kappa$. Assuming no local production of atoms, the steady-state spherically symmetric continuity equation is

$$
\frac{1}{r^{2}} \frac{d}{d r}\left(r^{2} n u\right)=-\kappa n
$$

For simplicity $\kappa$ and $u$ will be taken as constant. The solution to equation (12) is

$$
n(r)=n\left(r_{o}\right)\left(\frac{r_{o}}{r}\right)^{2} e^{-r / D\left(1-r_{o} / r\right)},
$$

where $r_{o}$ is the reference level (critical level in this case) and

$$
\begin{aligned}
D & =\frac{u}{\kappa} . \\
& =10^{5}\left(\frac{10^{-5} \mathrm{~s}^{-1}}{\kappa}\right)\left(\frac{u}{1 \mathrm{~km} \mathrm{~s}^{-1}}\right) \mathrm{km}
\end{aligned}
$$

is the depletion length scale. The value of $\kappa$ is determined by the total effective removal rate due to all nonthermal escape processes.

Based upon the escape rates calculated in $\S \mathrm{IV}$, we have made estimates for some of the rate coefficients for removal of atomic oxygen by the individual escape mechanisms.

$$
\begin{aligned}
\kappa_{\mathrm{eii}} & =2.8 \times 10^{-6} \mathrm{~s}^{-1} \text { (electron impact ionization) } \\
\kappa_{\mathrm{sp}} & =3.4 \times 10^{-4} \mathrm{~s}^{-1} \text { (sputtering) } \\
\kappa_{\mathrm{chex}} & =7.4 \times 10^{-6} \mathrm{~s}^{-1} \text { (charge exchange) }
\end{aligned}
$$

From $\S$ III it is seen that the relevant coronal length scale is $H$ which varies from about $10^{3} \mathrm{~km}$ at the critical level to $10^{4}$ $\mathrm{km}$ at the inner/outer Lagrangian points. For fast removal of atoms to significantly alter the density profile we must therefore have $D \leq 10^{3}$ to $10^{4} \mathrm{~km}$. We adopt a value of $u$ equal to the bulk flow velocity $u_{\text {flow }}=0.42 u_{s}$ at $r=r_{c}$. Escape due to electron impact ionization and charge exchange will therefore produce a depletion length scale $D \geq 3.0 \times 10^{4} \mathrm{~km}$, still large compared to $H\left(r_{c}\right)$ and a factor of 3 larger than $H\left(r_{L}\right)$. Sputtering, on the other hand, will produce a depletion length scale $\sim 1000 \mathrm{~km}$, comparable to the local thermal scale height. Thus sputtering may significantly alter the radial density variation in the corona, assuming that the full impact of the corotating plasma reaches to the exobase with no deflection.

The ram pressure of the magnetospheric wind flowing past Io will tend to distort the structure of Io's upper atmosphere. The ram pressure

$$
P_{\text {ram }} \sim n m(\delta u)^{2} \sim 1.1 \times 10^{-6} \text { dynes } \mathrm{cm}^{-2},
$$

where we have taken $n=1300 \mathrm{~cm}^{-3}$ and $m=16$ amu. By comparison the atmospheric gas pressure for model $\mathbf{B}$ $(P=n k T)$, is equal to the ram pressure at $n=7.8 \times 10^{6} \mathrm{~cm}^{-3}$ which occurs at $r=1.3 R_{\mathrm{Io}}$, i.e., $360 \mathrm{~km}$ below the critical level. A comet-like distortion of Io's atmosphere is therefore expected above this level, assuming no deviation or deceleration of plasma flow.

The large nonthermal escape rates calculated in these models imply substantial momentum transfer in the plasma torus/atmosphere interaction. As noted by Kumar (1979) the escape rates inferred for Io indicate mass loading of the torus above the Michel limit (Michel 1971). It is not clear if this limit holds for Io. Such large mass loading should not only decelerate the plasma but also cause substantial deflection of the flow. As seen in $\S$ IV the major culprit for producing large escape rates is atmospheric sputtering which occurs predominantly near the critical level. This assumes that the plasma actually reaches the critical level. If mass loading of the plasma is sufficient to deflect the plasma flow even one scale height above the critical level, very little actual atmospheric sputtering will occur. This would have the effect of decreasing the efficiency of the other nonthermal escape rates as well, lowering the total escape rate by at least a factor of 2 and possibly much more. It is clear that determining the plasma flow morphology near Io is crucial for calculating the atmospheric escape rate. 


\section{b) Observational Consequences}

Sodium is the most readily observed constituent near Io and thus provides a candidate tracer of atmospheric structure and escape processes. Unfortunately, the best spatial resolution of near-IoNa from Earth-based observations is approximately 1" or equivalently one Io diameter (Trauger 1984, 1985; Schneider et al. 1987). The best one can hope to infer from these observations is information on the gross structure of Io's extended neutral corona. The scale height of Io's atmosphere near the surface is much smaller than the available spatial resolution to permit near-surface structure determination. The simple models of Io's extended neutral corona presented in this paper provide a basis for comparison between observations of near-Io neutral clouds and theory.

The observed width of the high-velocity downstreamdirected $\mathrm{Na}$ jet seems to imply an extended source region of coronal $\mathrm{Na}$ in which charge exchange (C5) can occur. As can be seen from the model results the calculated source strength is strongly dependent upon the mixing ratios of $\mathrm{Na}^{+}$in the torus and $\mathrm{Na}$ in the corona and the critical level radius. Thus if one knows the torus abundance of $\mathrm{Na}^{+}, f_{\mathrm{Na}}\left(r_{c}\right)$, and the source strength, it is then possible to set a constraint on $r_{c}$. Since $f_{\mathrm{Na}}$ (torus) is poorly known it is only possible to infer a range for $r_{c}$. If the model of Io's corona in this paper approximates the real situation then it is likely, as indicated from a comparison of the model results and observations of $\mathrm{Na}$ near Io, that Io's critical level lies in the range $r_{c}=1.5$ to $2.0 r_{\mathrm{Io}}$. The source strength of the jet is consistent with reaction $\mathrm{C} 5$ generating the jet and a $1 \%$ abundance of $\mathrm{Na}$ at the critical level.

The Voyager PLS experiment electron temperature data contained a signature which has been tentatively identified to the passage of the torus plasma through a dense neutral corona around Io (Sittler and Strobel 1987). The observed electron temperature decrease from $T_{e}=5.5 \mathrm{eV}$ to a minimum value of $T_{e}=4.8 \mathrm{eV}$ at closest approach to Io may indicate a localized region of large ionization rates. Sittler and Strobel (1987) estimate that the observed $T_{e}$ drop indicates that thermal electrons penetrate the neutral corona down to approximately the exobase along magnetic flux tubes. A further theoretical study of the influence of a dense neutral corona on the longitudinal variation of torus properties is needed in order to determine observational consequences of this interaction.

Based on the preferred models A and B and the work of Summers (1985) for atmospheric composition down to Io's surface, the respective $O$ and $S$ radial column densities range from $(1-2) \times 10^{15} \mathrm{~cm}^{-2}$ and $(2-4) \times 10^{14} \mathrm{~cm}^{-2}$. The interaction of the atmosphere and the plasma torus should produce strong neutral emissions via electron impact excitation. The greatest uncertainties are how deeply the plasma penetrates the corona before being diverted around Io and how rapidly the electron temperature decreases as it penetrates the corona. The work of Sittler and Strobel (1987) suggests that plasma penetration and electron cooling does take place in the uppermost regions of Io's atmosphere.

The observations of Ballester et al. (1987) indicate hot electron interaction with large column densities of atoms near Io. If this is due to torus plasma penetration or to the presence of a dense hot ionosphere, a collisionally thick atmosphere is needed to produce the intensity of the observed emissions. As is seen from the models presented in this paper, the presence of a collisionally thick atmosphere, even if it is only $\sim 100 \mathrm{~km}$ thick, would imply an extensive thermal corona, and hence a region for significant torus/corona interaction that probably extends several $R_{\mathrm{Io}}$ from Io. This is consistent with observational data on the near-Io apparent width of the $\mathrm{Na}$ jets (Trauger 1984, 1985).

The distribution of $\mathrm{Na}$ as obtained by Schneider et al. (1987) has the same morphology as that of a thermal exosphere. The inferred radial density profile (see, e.g., model C in Schneider et al. 1987) is equivalent to a thermal exosphere with exobase at the surface and a $\mathrm{Na}$ mixing ratio of order 0.001 . Given the error bars in Schneider et al. (1987), their inferred density distribution is equivalent to model $\mathrm{C}$ in this paper. In view of the linearity of the radial density distribution to mixing ratio for a minor constituent, the Schneider et al. (1987) distribution is consistent with model $\mathrm{A}$ with a $\mathrm{Na}$ mixing ratio $\sim 5 \times 10^{-4}$ and model $\mathrm{B}$ with a $\mathrm{Na}$ mixing ratio $\sim 1 \times 10^{-3}$ (see Fig. 4). Thus the Schneider et al. (1987) inferred distribution is consistent with a thermal corona; however, with little constraint on the $\mathrm{Na}$ mixing ratio, it is difficult to constrain the exobase location with that distribution alone.

The magnitude of the calculated escape rates for the models of the thermal corona presented in this paper which include sputtering at the critical level are substantially larger than previously inferred torus supply rates. The fact that a thermal corona will be very extended suggests that possibly a major fraction of the escape is from regions above the critical level. The models presented here suggest that the interaction with the plasma torus above the critical level is substantial and can generate the necessary supply rate of neutrals to the torus even if no atmospheric sputtering (from the exobase) occurs. This would be consistent with a scenario in which torus plasma is deflected above the critical level. A strong interaction is in fact suggested by the observations of Ballester et al. (1987) and the theoretical work of Shemansky (1988).

The calculations of nonthermal escape rates in this paper assume full penetration of torus plasma to the exobase. As noted by McGrath and Johnson (1987), the calculated escape rates depend critically on the magnitude of the ion flux incident on the exobase region. Also, as we have shown, the structure and the escape rate from the thermal corona is sensitive to the degree of plasma deflection around the exobase. This is the key question regarding the interaction of Io's upper atmosphere and the plasma torus. The thermal corona model may only be applicable for a case in which plasma deflection occurs substantially above the exobase level. In such a case the calculated escape rates here represent upper limits. On the other hand, if the torus plasma impacts the exobase unattenuated and undeflected, the more accurate description of Io's neutral corona would be that of a sputter-generated nonthermal corona, and the interaction of such a corona with the plasma torus (Sieveka 1983; Sieveka and Johnson 1984, 1985, 1986; McGrath and Johnson 1987). In fact, models of the extended $\mathrm{Na}$ clouds around Io and along its orbit suggest that the nonthermal $\mathrm{Na}$ component near Io's exobase dominate over the thermal component by as much as possibly an order of magnitude (Smyth and Combi 1987, 1988a, b). These latter studies also suggest that nonthermal processes do play a major role in determining the radial structure of the neutral corona.

More accurate estimates of the escape and near Io massloading rates must await a better understanding of the plasma flow morphology around Io. However, it seems clear that due to Io's low gravity, the high atmospheric/exospheric temperature, and Io's location in a high-energy plasma environment, several distinct escape processes may be operative. Elucidating the precise way in which Io loses mass will 
depend to a large degree upon a detailed study of past and future observational results, and upon the development of theoretical models which can accurately describe the complex interactions which are probably occurring between Io's atmosphere and the plasma torus. To the extent that a thermal corona is a correct model of Io's extended atmosphere, model B with a $\mathrm{Na}$ mixing ratio $\sim 1 \times 10^{-3}$ represents the best description of a corona which is consistent with the $\mathrm{Na}$ jets observed by Trauger (1984) and the extended $\mathrm{Na}$ cloud detected by Schneider et al. (1987).

The authors thank W. H. Smyth, N. Schneider, E. Sittler, and G. Ballester for useful discussions on this topic. This work was supported by the National Aeronautics and Space Administration, contract NAGW-648 and the Voyager Mission.
Ballester, G. E., et al. 1987, Ap. J. (Letters), 319, L33.

Belcher, J. W. 1983, in Physics of the Jovian Magnetosphere, ed. A. J. Dessler (London: Cambridge), p. 68.

Brown, R. A. 1974, in Exploration of the Planetary System, ed. A. Woszcyk and C. Iwaniszerska (Dordrecht: Reidel), p. 527.

Brown, R. A. 1981, Ap. J., 244, 1072.

Brown, R. A., Pilcher, C. B., and Strobel, D. F. 1983, in Physics of the Jovian

Magnetosphere, ed. A. J. Dessler, p. 197.

Brown, R. A., Shemansky, D. E., and Johnson, R. E. 1983, Ap. J., 264, 309.

Brown, R. A., and Schneider, N. M. 1981, Icarus, 48, 519.

Chamberlain, J. W. 1963, Planet. Space Sci., 11,901.

Cheng, A. F. 1984, J. Geophys. Res., 89, 3939.

Dessler, A. 1980, Icarus, 44, 291.

Durrance, S. T., Feldman, P. D., and Weaver, H. A. 1983, Ap. J., 267, L125.

Goldberg, B. A., Garnau, G. W., and LaVoie, S. K. 1984, Science, 226, 512.

Haff, P. K., and Watson, C. C. 1979, J. Geophys. Res., 84, 8436.

Haff, P. K., Watson, C. C., and Yung, Y. L. 1981, J. Geophys. Res., 86, 6933.

Hunten, D. 1982, Planet. Space Sci., 30, 773.

1985, Geophys. Res. Letters, 12, 271

Ip, W.-H. 1982, Ap. J., 262, 780.

Johnson, R. E., and Strobel, D. F. 1982, J. Geophys. Res., 87, 385.

Kliore, A. J., Fjeldbo, G., Seidel, B. L., Sweetnam, D. M., Sespaulkis, T. T., and

Woiceshyn, P. M. 1975, Icarus, 24, 407.

Konowalow, D. D., Hirschfelder, J. O., and Linder, B. 1959, J. Chem. Phys., 31, 1575.

Kumar, S. 1979, Nature, 280, 758.

. 1980, Geophys. Res. Letters, 7, 9.

1982, J. Geophys. Res. 87, 1677.

1984, J. Geophys. Res., 89, 7399.

Kumar, S., and Hunten, D. 1982, in Satellites of Jupiter, ed. D. Morrison

(Tucson: University of Arizona Press), p. 782

McEwen, A. S., and Soderblom, L. A. 1983, Icarus, 55, 191.

\section{REFERENCES}

McGrath, M. A., and Johnson, R. E. 1987, Icarus, 69, 519.

Michel, F. C. 1971, Planet. Space Sci., 19, 1580.

Pilcher, C. B., Smyth, W. H., Combi, M. R., and Fertel, J. H. 1984, Ap. J., 287, 427.

Pollack, J. B., and Witteborn, F. C. 1980, Icarus, 44, 249.

Schneider, N. M., Hunten, D. M., Wells, W. K., and Trafton, L. M. 1987, Science, 238, 55 .

Shemansky, D. E. 1982, Ap. J., 242, 1266.

. 1988, J. Geophys. Res., 93, 1773.

Sieveka, E. M. 1983, Ph.D. thesis, University of Virginia.

Sieveka, E. M., and Johnson, R. E. 1982, Icarus, 51, 528.

-. 1984, Ap. J., 287, 418.

1985 , J.Geophys. Res. 90, 5327

1986, J. Geophys. Res., 91, 4608.

Sieveka, E. M., McGrath-Kinally, M., and Johnson, R. E. 1985, Bull. AAS, 17, 697.

Sittler, E. C., Jr., and Strobel, D. F. 1987, J. Geophys. Phys., 92, 5741.

Smirnov, B. M. 1965, Soviet Physics JETP, 20, 345.

Smith, R. A., and Strobel, D. F. 1985, J. Geophys. Res., 90, 9469

Smyth, W. H., and Combi, M. R. 1987, Geophys. Res. Letters, 14, 973.

. 1988a, Ap. J. Suppl., 66, 397. 1988 b, Ap. J., 328, 888 .

Smyth, W. H., and McElroy, M. B. 1977, Planet. Space Sci., 25, 415. 1978, Ap. J., 226, 336.

Smyth, W. H., and Shemansky, D. E. 1983, Ap. J., 271, 865.

Summers, M. E. 1985, Ph.D. thesis, California Institute of Technology.

Summers, M. E., Yung, Y. L., and Haff, P. K. 1983, Nature, 304, 710.

Trafton, L. 1975, Nature, 258, 690 .

. 1981, Ap.J., 247, 1125 .

Trauger, J. T. 1984, Bull. $A A S, 16,712$.

- 1985, Bull AAS, 17,697.

Watson, C. C. 1981, Proc. Lunar Planet Sci., 12B, 1569.

D. F. STRoBel: Department of Earth and Planetary Sciences, The Johns Hopkins University, Baltimore, MD 21218

Michael E. Summers: Mail Code 414.5, Space Science Division, Naval Research Laboratory, Washington, DC 20375

Frank Mills, J. T. Trauger, and Y. L. Yung: Mail Code 170-25, California Institute of Technology, Pasadena. CA 91125 